\title{
Erratum to: Drivers and mechanisms for enhanced summer monsoon precipitation over East Asia during the mid-Pliocene in the IPSL-CM5A
}

\author{
Yong Sun ${ }^{1,2}$ - Tianjun Zhou ${ }^{1,2}$ - Gilles Ramstein ${ }^{3}$ - Camille Contoux ${ }^{4}$. \\ Zhongshi Zhang ${ }^{5,6}$
}

Published online: 29 August 2015

C) Springer-Verlag Berlin Heidelberg 2015

\section{Erratum to: Clim Dyn \\ DOI 10.1007/s00382-015-2656-4}

There are some mathematic expression problems in this paper due to proofing errors. Now I write an erratum to correct the mathematic derivation as listed in the table. In addition, $p$ should have been subscript in all the $C p$ relevant terms (i.e., $C p$ ).

The online version of the original article can be found under doi:10.1007/s00382-015-2656-4.

Tianjun Zhou

zhoutj@lasg.iap.ac.cn

1 State Key Laboratory of Numerical Modeling for Atmospheric Sciences and Geophysical Fluid Dynamics, Institute of Atmospheric Physics, Chinese Academy of Sciences, Beijing 100029, China

2 Joint Center for Global Change Studies (JCGCS), Beijing 100875, China

3 Laboratoire des Sciences du Climat et de l'Environnement/ IPSL, CEA-CNRS-UVSQ, Gif-Sur-Yvette, France

4 Aix-Marseille Université, CNRS, IRD, CEREGE UM34, Aix-En-Provence, France

5 Bjerknes Centre for Climate Research, Uni Research Climate, Allégaten 70, 5007 Bergen, Norway

6 Nansen-Zhu International Research Centre, Institute of Atmospheric Physics, Chinese Academy of Sciences, Beijing 100029, China

\begin{tabular}{|c|c|c|}
\hline Position & Incorrect & Correct \\
\hline $\begin{array}{l}\text { The left half page } \\
\text { P9 Line } 12\end{array}$ & $-\langle-C p \overline{V \cdot \nabla T}\rangle$ & $-\left\langle C_{p} \overline{V \cdot \nabla \mathrm{T}}\right\rangle$ \\
\hline $\begin{array}{l}\text { The left half page } \\
\text { P9 Line } 13\end{array}$ & $-\left\langle-L_{v} \overline{V \cdot \nabla q}\right\rangle$ & $-\left\langle L_{v} \overline{V \cdot \nabla q}\right\rangle$ \\
\hline $\begin{array}{l}\text { The right half page } \\
\text { P9 Line } 2\end{array}$ & $-\left\langle-L_{v} \overline{V \cdot \nabla q}\right\rangle$ & $-\left\langle L_{v} \overline{V \cdot \nabla q}\right\rangle$ \\
\hline $\begin{array}{l}\text { The right half page } \\
\text { P9 Line } 7\end{array}$ & $-\langle-C p \overline{V \cdot \nabla \mathrm{T}}\rangle$ & $-\left\langle C_{p} \overline{V \cdot \nabla \mathrm{T}}\right\rangle$ \\
\hline $\begin{array}{l}\text { The right half page } \\
\text { P9 Line } 12\end{array}$ & $-\langle-C p \overline{V \cdot \nabla \mathrm{T}}\rangle$ & $-\left\langle C_{p} \overline{V \cdot \nabla \mathrm{T}}\right\rangle$ \\
\hline $\begin{array}{l}\text { The right half page } \\
\text { P9 Line } 16\end{array}$ & $-\langle-C p \overline{V \cdot \nabla \mathrm{T}}\rangle$ & $-\left\langle C_{p} \overline{V \cdot \nabla \mathrm{T}}\right\rangle$ \\
\hline $\begin{array}{l}\text { The left half page } \\
\text { P10 Line } 4\end{array}$ & $-\langle-C p \overline{V \cdot \nabla \mathrm{T}}\rangle$ & $-\left\langle C_{p} \overline{V \cdot \nabla \mathrm{T}}\right\rangle$ \\
\hline $\begin{array}{l}\text { The left half page } \\
\text { P10 Line } 6\end{array}$ & $-\left\langle-L_{v} \overline{V \cdot \nabla q}\right\rangle$ & $-\left\langle L_{v} \overline{V \cdot \nabla q}\right\rangle$ \\
\hline $\begin{array}{l}\text { The left half page } \\
\text { P10 Line } 9\end{array}$ & $-\langle-C p \overline{V \cdot \nabla \mathrm{T}}\rangle$ & $-\left\langle C_{p} \overline{V \cdot \nabla \mathrm{T}}\right\rangle$ \\
\hline $\begin{array}{l}\text { The left half page } \\
\text { P10 Line } 14\end{array}$ & $-\langle-C p \overline{V \cdot \nabla \mathrm{T}}\rangle$ & $-\left\langle C_{p} \overline{V \cdot \nabla \mathrm{T}}\right\rangle$ \\
\hline $\begin{array}{l}\text { The left half page } \\
\text { P10 Line } 17\end{array}$ & $-\langle-C p \overline{V \cdot \nabla \mathrm{T}}\rangle$ & $-\left\langle C_{p} \overline{V \cdot \nabla \mathrm{T}}\right\rangle$ \\
\hline $\begin{array}{l}\text { The right half page } \\
\text { P10 Line } 1\end{array}$ & $-\langle-C p \overline{V \cdot \nabla \mathrm{T}}\rangle$ & $-\left\langle C_{p} \overline{V \cdot \nabla \mathrm{T}}\right\rangle$ \\
\hline $\begin{array}{l}\text { The right half page } \\
\text { P10 Line } 19\end{array}$ & $-\langle-C p \overline{V \cdot \nabla \mathrm{T}}\rangle$ & $-\left\langle C_{p} \overline{V \cdot \nabla \mathrm{T}}\right\rangle$ \\
\hline $\begin{array}{l}\text { The right half page } \\
\text { P11 Line } 14\end{array}$ & $-\left\langle-C p \overline{V^{*}} \cdot\left[\overline{\partial T_{y}}\right]\right\rangle$ & $-\left\langle C_{p} \overline{V^{*}} \cdot\left[\overline{\partial T_{y}}\right]\right\rangle$ \\
\hline $\begin{array}{l}\text { The left half page } \\
\text { P12 Line } 5\end{array}$ & $-\left\langle-C p \overline{V^{*}} \cdot\left[\overline{\partial T_{y}}\right]\right\rangle$ & $-\left\langle C_{p} \overline{V^{*}} \cdot\left[\overline{\partial T_{y}}\right]\right\rangle$ \\
\hline $\begin{array}{l}\text { The right half page } \\
\text { P12 Line } 8\end{array}$ & $-\left\langle-C p \overline{V^{*}} \cdot\left[\overline{\partial T_{y}}\right]\right\rangle$ & $-\left\langle C_{p} \overline{V^{*}} \cdot\left[\overline{\partial T_{y}}\right]\right\rangle$ \\
\hline $\begin{array}{l}\text { The right half page } \\
\text { P12 Line } 11\end{array}$ & $-\left\langle-C p \overline{V^{*}} \cdot\left[\overline{\partial T_{y}}\right]\right\rangle$ & $-\left\langle C_{p} \overline{V^{*}} \cdot\left[\overline{\partial T_{y}}\right]\right\rangle$ \\
\hline $\begin{array}{l}\text { The right half page } \\
\text { P17 Line } 53\end{array}$ & $-\left\langle-C p \overline{V^{*}} \cdot\left[\overline{\partial T_{y}}\right]\right\rangle$ & $-\left\langle C_{p} \overline{V^{*}} \cdot\left[\overline{\partial T_{y}}\right]\right\rangle$ \\
\hline $\begin{array}{l}\text { The right half page } \\
\text { P18 Line } 2\end{array}$ & $-\left\langle-C p \overline{V^{*}} \cdot\left[\overline{\partial T_{y}}\right]\right\rangle$ & $-\left\langle C_{p} \overline{V^{*}} \cdot\left[\overline{\partial T_{y}}\right]\right\rangle$ \\
\hline
\end{tabular}

